\title{
Entwicklung eines neuronalen Netzwerks als Basis zur automatisierten Holzartenerkennung
}

\author{
A. Bernöcker ${ }^{1}$, N. Leiter ${ }^{1}$, M. Wohlschläger ${ }^{1}$, M. Versen ${ }^{1}$
}

\section{Zusammenfassung}

Holz ist ein vielseitig einsetzbarer nachwachsender Rohstoff. Neben seinem wirtschaftlichen Nutzen ist er für den Erhalt des Klimas unersetzlich. Eine sortenreine Sortierung für die Weiterverarbeitung von Altholz spielt für einen ressourcenschonenden Umgang eine wichtige Rolle. Um das Potenzial eines neuronalen Netzwerks basierend auf Messdaten der bildgebenden Fluoreszenzabklingzeitmessung für die Altholzsortierung aufzuzeigen, wurden zwei unterschiedliche Klassifikationsansätze auf Basis der Programmiersprache Python gewählt. Die Ergebnisse zeigen, dass die bildbasierte Klassifizierung der Holzart mit einer Genauigkeit von 47,36 \% noch ausbaufähig ist. Eine datenbasierte Klassifizierung der Holzart mit einer Identifikationsgenauigkeit von 98,28 \% ist dagegen vielversprechend.

\section{Stichwörter}

FD-FLIM, Fluoreszenz, Fluoreszenzabklingzeit, Holzart, Neuronales Netz, Klassifizierung

\section{Einleitung}

Holz wird in der Wirtschaft vielseitig genutzt wird und kann in Deutschland sowohl stofflich als auch energetisch verwertet werden. Für einen ressourcenschonenden Umgang mit dem Primärrohstoff Holz, wird derzeit zunehmend an der Wiederverwendbarkeit von Altholz geforscht, da allein in Deutschland das jährliche Marktvolumen ca. 10 Millionen Tonnen Altholz beträgt [1]. Die Qualität des Altholzes spielt bei der Wiederverwendung eine wichtige Rolle und wird deshalb in vier Altholzkategorien unterschieden: A1 für unbehandeltes Altholz, A2 für Altholz ohne halogenorganische Verbindungen (wie PVC) oder mit Holzschutzmittel behandeltes Altholz, A3 für mit PVC aber ohne Holzschutzmittel behandeltes Altholz und A4, bei dem es sich um kontaminiertes Altholz wie beispielsweise Bahnschwellen, Fenster oder Türen handelt [2]. Derzeit wird das Altholz nach der Altholzverordnung durch Sichtkontrolle zu sortiert [2]. Eine zuverlässige und automatisierte Technik konnte noch nicht eingeführt werden. Gegenwärtig werden hauptsächlich zwei Techniken zur Identifizierung von Holz erforscht: Die Nahinfrarot-Spektroskopie und die Röntgenfluoreszenz-Spektroskopie. Nach Mauruschat et. al. ist es möglich Holzarten mit Nahinfrarot-Spektroskopie zu identifizieren [3]. Die Erkennung wird jedoch durch die Überlagerung des Spektrums mit dem Absorptionsband des variablen Feuchtigkeitsgehalts im Holz erschwert. Die zweite Methode zur Identifizierung von Holz ist die Röntgenfluoreszenz-Spektroskopie [4]. Diese Methode ist nicht geeignet für eine automatisierte Holzidentifizierung, da nur Schadstoffe wie Schwermetalle mit hohen Ordnungszahlen identifiziert werden können. Somit besteht weiterhin der Bedarf an einer automatisierten Sortierungstechnik für Altholz. Im Laufe der Vorarbeiten konnte bereits mit Hilfe des Frequency-Domain Fluorescence Lifetime Imaging Microscopy (FD-FLIM) Verfahrens nachgewiesen werden, dass die Identifikation der

1 Technische Hochschule Rosenheim, Hochschulstr. 1, 83024 Rosenheim 
Holzart und Altholzkategorie aufgrund ihrer charakteristischer Fluoreszenzabklingzeiten möglich ist [5]. Hierbei wurde allerdings eine manuelle Klassifizierung anhand definierter Parameter durchgeführt. Um die manuelle Klassifizierung abzulösen und das Verfahren weiter zu automatisieren, wird der Einsatz eines neuronalen Netzwerks untersucht, das mit den Messdaten der FD-FLIM Messungen trainiert wird. Hierzu werden zwei Lösungsansätze in der Programmiersprache Python verfolgt: eine bildliche Klassifikation durch Merkmalserkennung und eine datenbasierte Klassifikation durch statistische Methoden. Die Klassifizierung der Holzart steht dabei im Vordergrund.

\section{Stand der Technik}

Um eine neue Methode der Holzartenerkennung vorzustellen, wird zunächst die Theorie der FDFLIM (frequency domain fluorescence lifetime microscopy) Technik sowie die Grundlagen künstlicher neuronalen Netzwerken erklärt.

\subsection{Die FD-FLIM Messtechnik}

Fluoreszenz beschreibt die spontane Emission von Licht nach der Anregung eines Materials mit einem Lichtimpuls. Die Fluoreszenzabklingzeit dient in der Fluorometrie zur Unterscheidung verschiedener Fluorophore und liefert Informationen über die chemische Umgebung eines Fluorophors. Die Fluoreszenzabklingzeit gibt die Zeit an, die ein Molekül bei der Fluoreszenz in einem angeregten Zustand bleibt, bevor es ein Photon emittiert und damit in den Grundzustand zurückkehrt. Die Abklingkurve verläuft exponentiell und konvergiert gegen Null. Auf der Grundlage der Systemtheorie kann die Fluoreszenzanregung nicht nur durch einen Anregungspuls (Dirac-Puls) im Zeitbereich erfolgen, sondern auch durch eine Testfunktion wie einer Sinus- oder Rechteckschwingung mit einer bestimmten Modulationsfrequenz $\omega$. Das Fluoreszenzsignal folgt der sinusförmigen Anregung mit gleicher Frequenz phasenverschoben um den Winkel $\varphi$. Darüber hinaus ist die Fluoreszenzschwingung in ihrer Amplitude gedämpft und in ihrem Gleichstromwert verschoben. In Abbildung 1 sind die Amplitude der Anregung B, die Amplitude der Fluoreszenzemission b, der Gleichstromwert der Anregung A und der Gleichstromwert der Fluoreszenzemission a sowie die Phasenverschiebung $\varphi$ markiert [6].

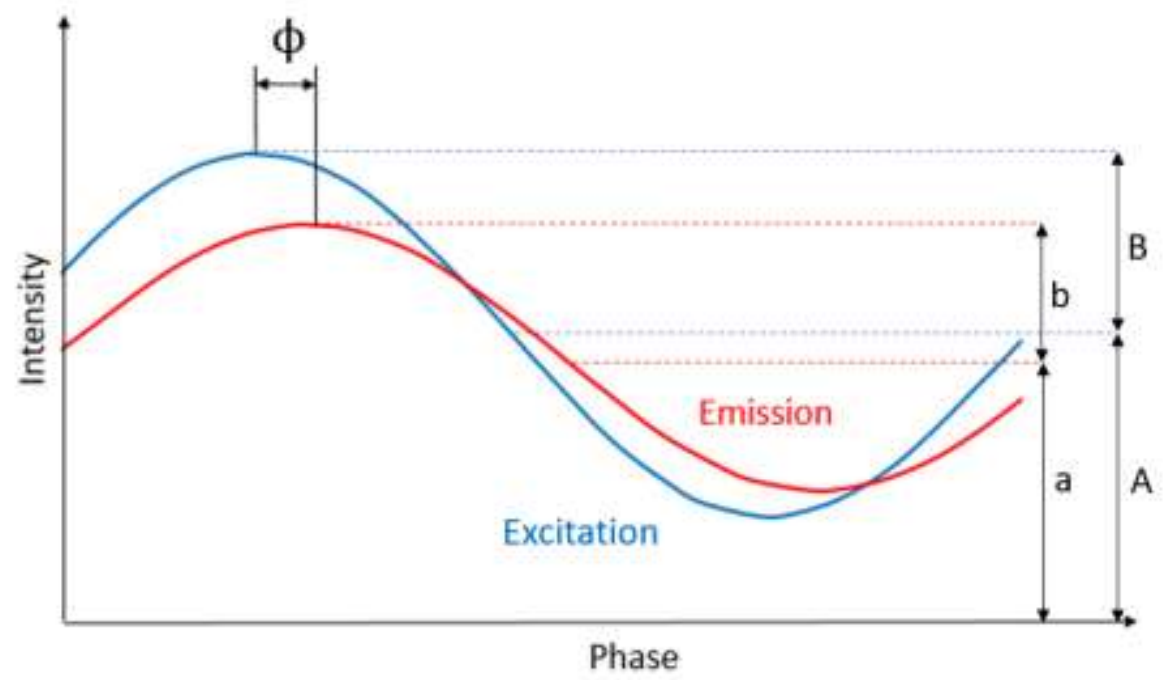

Abbildung 1: Darstellung der Phasenverschiebung $\varphi$, der Amplitudendämpfung B, b und der Gleichwertverschiebung A, a des Fluoreszenzsignals

Die Berechnung der Modulationstiefe $M$ erfolgt über die Differenz der Amplitude und der Gleichstromwerte [6]: 


$$
M=\left(\frac{b}{a}\right) /\left(\frac{B}{A}\right)
$$

Die Modulationstiefe M und die definierte Modulationsfrequenz $\omega$ lassen auf die modulationsabhängige Fluoreszenzabklingzeit $\tau_{M}$ schließen [6]:

$$
\tau_{M}=\frac{\sqrt{\frac{1}{M^{2}}-1}}{\omega}
$$

Mit der festgelegten Modulationsfrequenz $\omega$ kann die Phasenverschiebung $\varphi$ zur Berechnung der phasenabhängigen Fluoreszenzabklingzeit $\tau_{P h}$ verwendet werden [6]:

$$
\tau_{P h}=\frac{1}{\omega} * \tan (\varphi)
$$

Diese theoretischen Grundlagen bilden die Basis der FD-FLIM Kamera Technik. Der in [5] evaluierte Messaufbau mit einer FD-FLIM Kamera bestimmt die Intensität, die Phasenverschiebung, die Modulationstiefe, die Phasenabklingzeit und die Modulationsabklingzeit einer Holzprobe, welche in Form von ND2 Dateien vorliegen. Bei ND2 Dateien handelt es sich um multidimensionale Bildaufnahmen, welche 103,8 MB groß sind und aus fünf Ebenen bestehen. Jede Ebene bildet eine Matrix mit der Dimension von 1008x1004 Pixeln von den entsprechenden Kenngrößen (Intensität, Phasenverschiebung, Modulationstiefe, phasenabhängige Fluoreszenzabklingzeit und modulationsabhängige Fluoreszenzabklingzeit). Zudem werden Information (Metadaten) über die verwendete Kamera wie der Name, die Temperatur bei der Aufnahme oder die Belichtungszeit gespeichert.

\subsection{Künstliche Neuronale Netzwerke}

Künstliche neuronale Netzwerke (KNN) sind dem menschlichen Gehirn nachempfunden und werden für maschinelles Lernen und künstliche Intelligenz eingesetzt. Die Nutzung von KNN ist speziell im Bereich von Big Data oder auch im Bereich der Bilderkennung weit verbreitet. In einem künstlichen Neuron werden die Signale des vorgelagerten Neurons verstärkt oder abgeschwächt. Eine Propagierungsfunktion wandelt die gewichteten Signale in einen Minimal- oder Maximalwert aus den Summen der Eingangssignale. Eine Aktivierungsfunktion berechnet anschließend den Zustand der Neuronen [7].

Da die Probleme der Regression und Klassifikation in der Informatik häufig vorkommen, gibt es hierfür sogenannte Frameworks (vorgefertigte Programmierstrukturen) wie Tensorflow Keras oder SciKit Learn. Tensorflow Keras ist eine von Google bereitgestellte Abstraktionsbibliothek, welche den Vorteil hat, dass Nutzer flexibel und im Baukastenprinzip KNN erstellen können. Zusätzlich bietet Tensorflow Keras eine große Auswahl an bereits vortrainierten Netzwerken [8]. SciKit Learn ist eine freie Softwarebibliothek für maschinelles Lernen auf der Basis von Python [9]. Für die Programmierung von neuronalen Netzwerken werden Hyperparameter verwendet, die vor dem Lernprozess festgelegt werden. Die Hyperparameter beeinflussen das Netzwerk hinsichtlich der Effizienz und Leistung. Eine optimale „Learning Rate“ führt zu guten Ergebnissen bei niedrigen Lernzeiten. Das Trainieren von neuronalen Netzwerken wird mit Epochen durchgeführt. Eine Epoche stellt dabei einen Zyklus dar, bei dem das neuronale Netz einmal mit allen Daten trainiert wurde. Um die Gefahr des „Overfitting“, also das „Auswendig Lernen“ des Netzes beim Trainieren des Netzwerks zu verkleinern, wird die Callback Funktion „Early Stopping“ definiert, welche das Training beendet, sobald der Fehler der Ergebnisse nach einer zuvor festgelegten Anzahl von Epochen stagniert [10].

Im Rahmen der Untersuchungen wurden zwei Arten von künstlichen Neuronalen Netzwerken gegenübergestellt: ein Convolutional Neural Network (CNN) für eine bildbasierte Klassifizierung mittels 
Tensorflow Keras und ein Multilayer Perceptron Network (MLP) für eine datenbasierte Klassifizierung mittels SciKit Learn.

Das CNN ist ein mehrschichtiges feedforward-Netz und gehört zu der Klasse der deepneural-Netze. Das CNN beinhaltet einen Kodierungsblock und einen Prädikationsblock. Im Falle eines Farbbildes, stehen die drei Dimensionen, also Höhe, Breite und Tiefe des Bildes jeweils in Form einer Matrix der „Convolutional Layer“ des Kodierungsblocks zur Verfügung. Zweidimensionales Arrays decken als Filter über das Faltungsprodukt Merkmale auf, wobei teilweise mehrere Filter kombiniert werden. Die Aktivierungsfunktion „Rectified Linear Unit Funktion“ setzt nach jeder Faltung negative Werte auf 0, sodass eine Nichtlinearität eingeführt wird. Es folgt ein „Pooling Layer“ zur Reduzierung der Dimensionalität über einen Filter ohne Gewicht. Üblich ist hier ein sogenannter „Max Pooling Layer“ für eine Weitergabe der maximalen Werte der Pixel. Der Prädikationsblock des CNN besteht aus einem fullyconnected-Netz und hat als Ziel die Ausgabe der Wahrscheinlichkeitsverteilung der Klassen über die Aktivierungsfunktion „Softmax“ [11].

Ein Perceptron ist ein Rechenmodell, welches die Lösung binärer Klassifikationsprobleme vereinfacht. Der Bias ermöglicht dabei dem Netz die Richtungslenkung der Entscheidungstrennlinie, was zu einem schnellen und qualitativ hochwertigen Trainingsprozess führt. Wie das CNN ist auch das MLP ein feedforward-Netz und zählt zu den artifical-neural-Netzen. Während der „Forward Propagation“ werden die Daten von einem „Input Layer“ erfasst. In mehreren „Hidden Layer“ erfolgt der Lernprozess, indem die Gewichte und der Bias berechnet werden und im Output Layer die daraus folgende Fehlerquote bestimmt wird. In der „Backward Propagation“ erfolgt durch die Verarbeitung dieser Informationen inklusive der Fehlerquote eine Neujustierung der Gewichte. Dieser Vorgang wird für einen minimal Fehler wiederholt [12].

\section{Methodisches Vorgehen}

Das gesamte Projekt wurde in der Programmiersprache Python entwickelt. Verwendet wurde die Python Version 3.8 mit der Entwicklungsumgebung PyCharm Community Edition 2019.3.3

\subsection{Die Holzproben}

Es werden standardisierte Holzproben der Holzarten Ahorn, Buche, Eiche, Fichte, Lärche und Kiefer verwendet. Die Proben werden an drei Seiten markiert, welche die Schnittrichtung radial, tangential oder Hirnholz bezeichnet. Jede der drei Seiten einer Holzprobe wird mittels FD-FLIM jeweils 16 mal mit den Wellenlänge von $405 \mathrm{~nm}$ und $488 \mathrm{~nm}$ vermessen, und die Daten werden in Form von ND2Dateien gespeichert. Das Vorgehen bei den Messungen ist in [5] detailliert dargestellt.

\subsection{Die Datenvorbereitung}

Die generierten Daten werden über das Python Package „nd2reader“ eingelesen. Aus jeder ND2-Datei werden fünf Matrizen gemäß den fünf Lagen (Intensität, Phasenverschiebung, Modulationstiefe, phasenabhängige Fluoreszenzabklingzeit und modulationsabhängige Fluoreszenzabklingzeit) generiert und im Anschluss als TIF Bild mit der jeweiligen Lagenbezeichnung in einem Ordner gespeichert.

Für die bildliche Klassifizierung und die Verwendung des CNN werden die erstellten TIF Dateien für jede Holzart über das Python Package „tifffile“ geöffnet. Die Bilder werden anschließßend für die spätere Betrachtung in ein RGB Bild umgewandelt und abgespeichert. Dieser Schritt ändert die Dimensionalität der Bilder von 1008x1004 auf 1008x1004x3. Für die Normierung der Intensität werden die Matrizen der Intensität mit der Belichtungszeit multipliziert. Um einen größeren Datensatz zu generieren, wurde eine Kombination aus phasenabhängiger Abklingzeit und Intensität kreiert. Zusätzlich werden die Trainingsbilder jeweils um 90, 180 und 270 Grad gedreht. Somit entsteht ein Datensatz aus 
1404 Trainingsdaten, 144 Validierungsdaten und 84 Testdaten, was einer Gesamtbilderanzahl von 1632 entspricht.

Zur Umsetzung des zweiten Lösungsansatzes wurde ein MLP verwendet, das eine tabellarische Dateneingabe benötigt. Daher werden die ND2 Dateien über eine selbst erstellte Python Funktion geöffnet und die Matrizen der phasenabhängigen und der modulationsabhängigen Abklingzeit extrahiert. Aus den beiden Matrizen wird jeweils die Standardabweichung und der Mittelwert berechnet und über das Python Package „pandas“ als Tabelle gespeichert. Der Datensatz enthält 288 Messungen, der aus 48 Messungen der sechs Holzarten Ahorn, Buche, Eiche, Fichte, Lärche und Kiefer besteht. Aufgrund der Einteilung von 80:20 entsteht ein Trainingsdatensatz von 230 Messungen und ein Testdatensatz von 58 Messungen.

\subsection{Die bildbasierten Klassifizierung}

Die RGB Dateien werden mit einer Funktion wie folgt aufgeteilt: 60 Prozent Trainingsdaten, 25 \% Validierungsdaten und $15 \%$ Testdaten. Mit der von Tensorflor Keras bereitgestellten „Proprocessing“ Funktion des vortrainierten „MobileNet“ wird eine Normalisierung und Aufbereitung der Bilder durchgeführt. Nachdem die Bilder optimal vorbereitet wurden, wird das Netz über die Funktion „build_net“ erstellt. Zu Beginn der Netzwerkerstellung wird dazu das vortrainierte „MobileNet“ von Tensorflow Keras verwendet und als Basis-Model gespeichert. Im Basis-Modell werden die bereits abgestimmten Gewichte des Netzes eingefügt. Mit „Include_top=False“ werden die am Ende verwendeten fully-connected-Layer entfernt, da diese im Anschluss an das Transferlernen neu erstellt werden. Zudem muss dem Netz der benutzerdefinierte Input-Shape mitgeteilt werden. Das Model wird kompiliert und die Optimierungsfunktion „Adam“ mit einer Lernrate von 1e-4 wird an das Modell übergeben. Um „Overfitting“ zu vermeiden, wird ein „Early Stopping“ definiert. Insgesamt wird das Netz 50 Epochen trainiert mit einer „Batchsize“ von 10.

\subsection{Die datenbasierten Klassifizierung}

Für den Ansatz der datenbasierten Klassifikation wurde der „MLPClassifier“ von SciKit Learn verwendet. Der „MLPClassifier“ ist im Vergleich zu den vortrainierten Bildklassifizierungs-Modellen deutlich schneller zu trainieren und einfacher zu verwenden. Die vorbereite Datentabellen wird als „Pandas Dataframe“ eingelesen. Die Holzart (Klasse), welche dem Netz als Label dient, ist als String in der Tabelle hinterlegt. Ein Teil des „Preprocessing“ besteht darin, über einen „LabelEncoder“ von SciKit Learn die Klasse in einen nummerischen Wert umzuwandeln. Bei 6 Klassen erhält man somit eine Klassenbezeichnung von 0 bis 5. Mit Hilfe des „StandardScaler“ von SciKit Learn werden die zahlenbasierten Daten normalisiert und anschließend in Eingabedaten und Zieldaten aufgeteilt. Die Eingabedaten bestehen aus jeweils dem Mittelwert und der Standardabweichung der Intensität, der phasenabhängigen Abklingzeit und der modulationsabhängigen Abklingzeit. Als Zieldaten dienen die Klassen, die das Netz ausgeben soll. Die Eingabe- und Zieldaten werden jeweils 80:20 in Trainings- und Testdaten geteilt. Nachfolgend wird der „MLPClassifier“ erzeugt. Die „GridSearch“ Funktion durchläuft die möglichen Parameterkombination, trainiert das Netz damit und gibt am Ende aus, welche Kombination das beste Ergebnis geliefert hat. Die Kombination wird im Anschluss dem „MLPClassifier“ übergeben, sodass das Netz trainiert werden kann. 


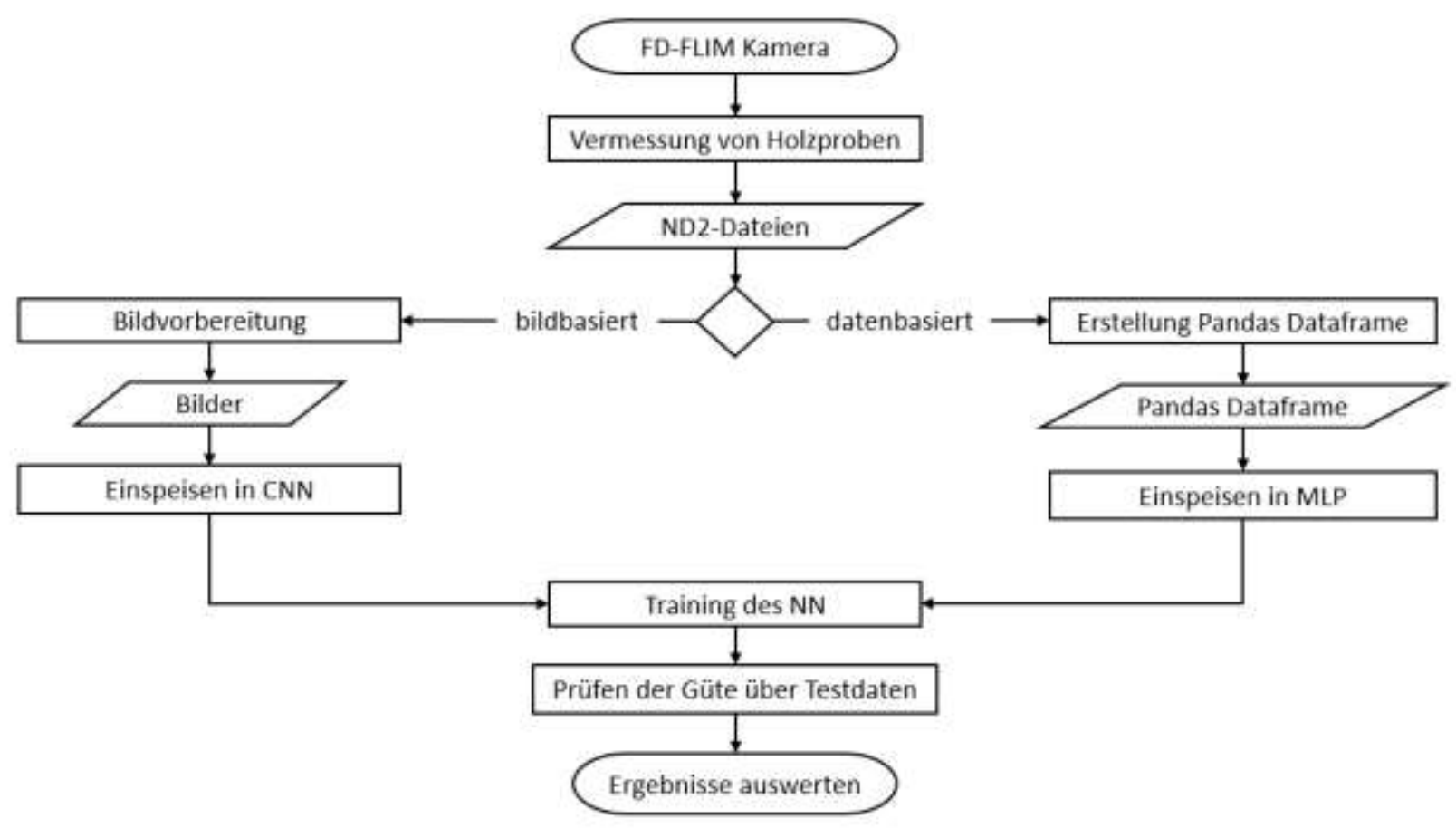

Abbildung 2: Flussdiagramm zur Übersicht des methodischen Vorgehens

\section{Ergebnisse und Fazit}

\subsection{Die Testergebnisse des CNN}

In Abbildung 2 ist das Testergebnis des CNN bei einer Anregungswellenlänge von $405 \mathrm{~nm}$ mit Hilfe einer Konfusionsmatrix dargestellt.

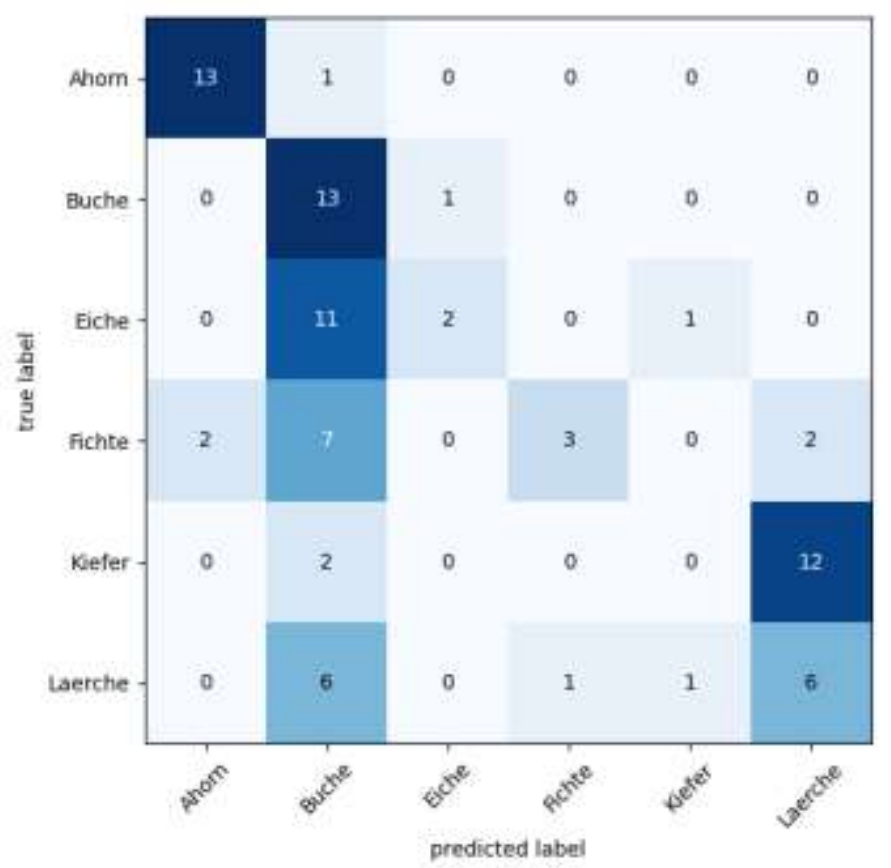

Abbildung 3: Konfusionsmatrix der bildbasierten Klassifizierung von Holzarten bei einer Anregung von $405 \mathrm{~nm}$ 
Anhand der Diagonalen in der Konfusionsmatrix ist zu erkennen, dass bei einer Anregungswellenlänge von 405 nm wird die Holzart für 37 von 84 Proben korrekt bestimmt wurden. Die Proben wurden mit einer Erkennungsgenauigkeit von 44,05\% zugeordnet. Ahorn und Buche wurden 13 von 14mal richtig erkannt und schnitten in diesem Test am besten ab. Lärche wurde lediglich 6 von 14-mal erkannt.

Abbildung 3 zeigt analog das Testergebnis des CNN bei einer Anregungswellenlänge von $488 \mathrm{~nm}$.

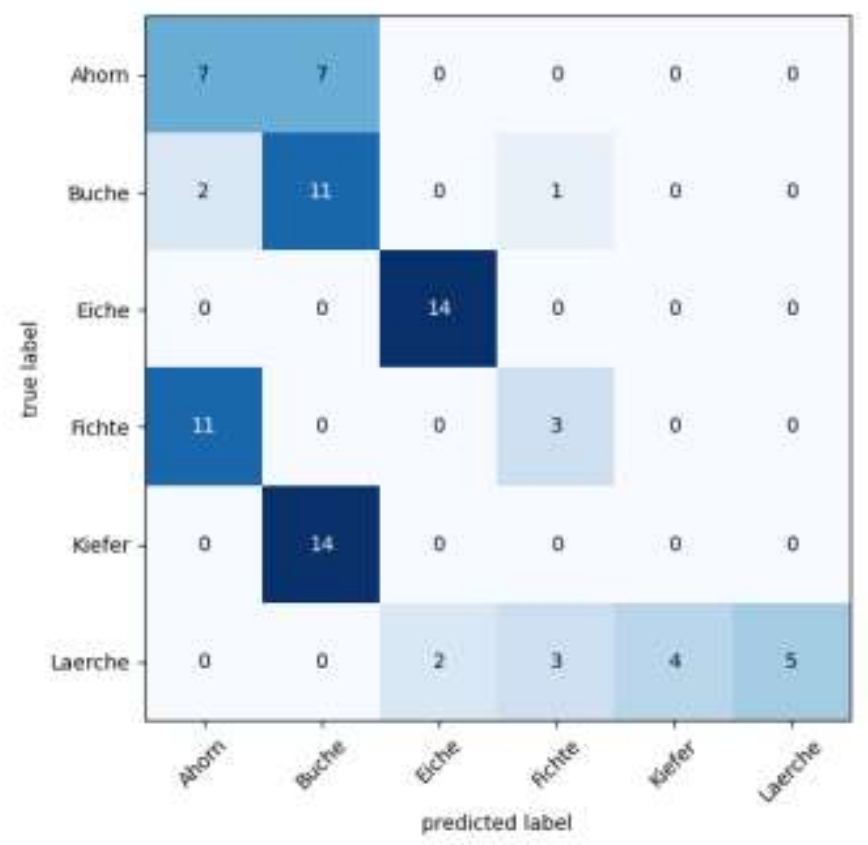

Abbildung 4: Konfusionsmatrix der bildbasierten Klassifizierung von Holzarten bei einer Anregung von $488 \mathrm{~nm}$

Bei einer Anregungswellenlänge von 488nm wurde die Holzart für 40 von 84 Proben korrekt bestimmt. Dies entspricht 47,62\% Erkennungsgenauigkeit. Eiche wurde 14 von 16-mal richtig erkannt und schnitt in diesem Test am besten ab. Die Identifikation der Holzarten Fichte, Kiefer und Lärche stellte sich hierbei noch sehr problematisch dar. Lediglich 3-mal wurde Fichte korrekt erkannt, Lärche 5-mal und Kiefer wurde stets falsch zugewiesen.

\subsection{Die Testergebnisse des MLP}

In Abbildung 4 ist das Testergebnis des MLP bei einer Anregungswellenlänge von $405 \mathrm{~nm}$ in einer Konfusionsmatrix dargestellt.

Die Matrix zeigt, dass bei einer Anregungswellenlänge von 405nm die Holzart für 57 von 58 Proben korrekt bestimmt. Dies entspricht einer Genauigkeit von 98,28 \%. Ahorn, Eiche, Fichte und Kiefer wurden jeweils 10 von 10-mal und Buche 9-mal richtig erkannt. Lärche wurde einmal fälschlicherweise Fichte zugeordnet. 


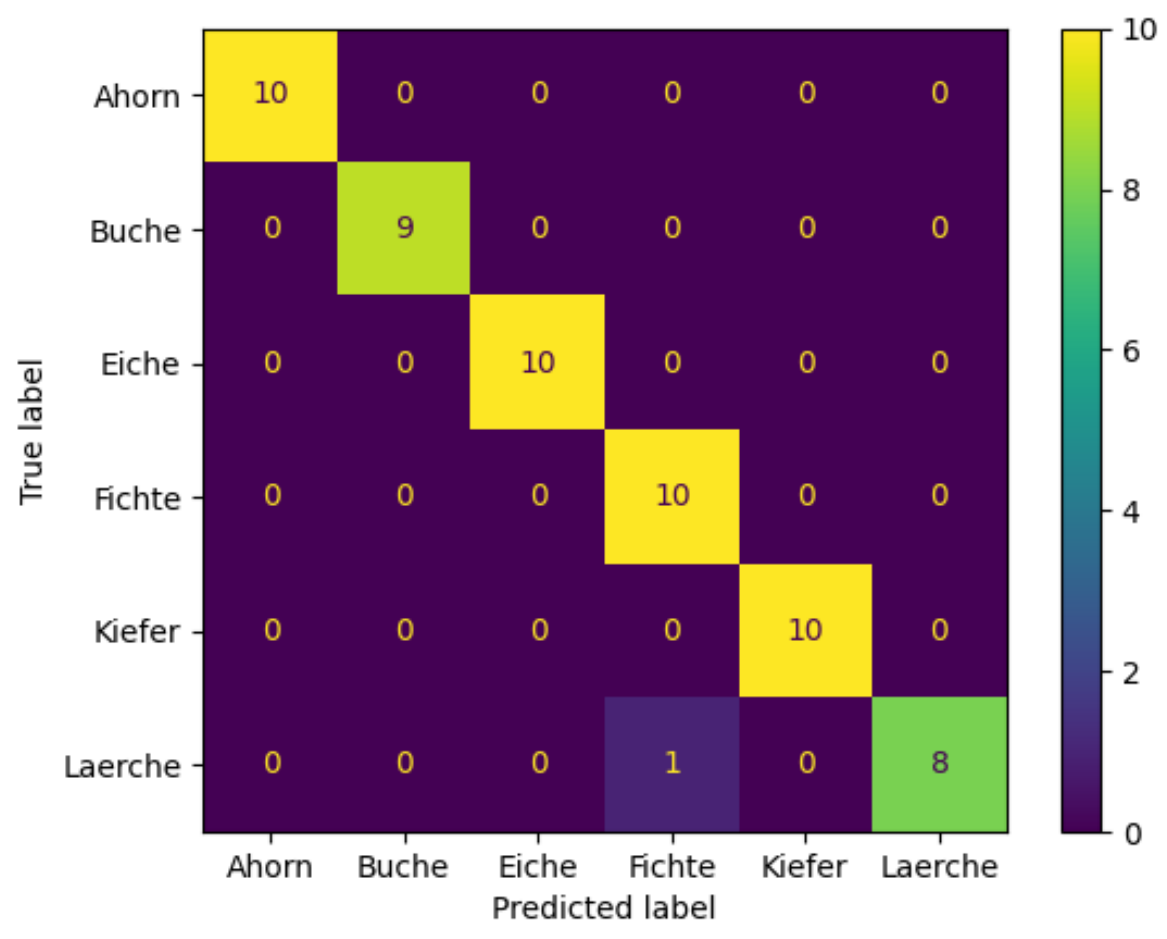

Abbildung 5: Konfusionsmatrix der datenbasierten Klassifizierung von Holzarten bei einer Anregung von $405 \mathrm{~nm}$

In Abbildung 5 ist analog das Testergebnis des MLP bei einer Anregungswellenlänge von $488 \mathrm{~nm}$ dargestellt.

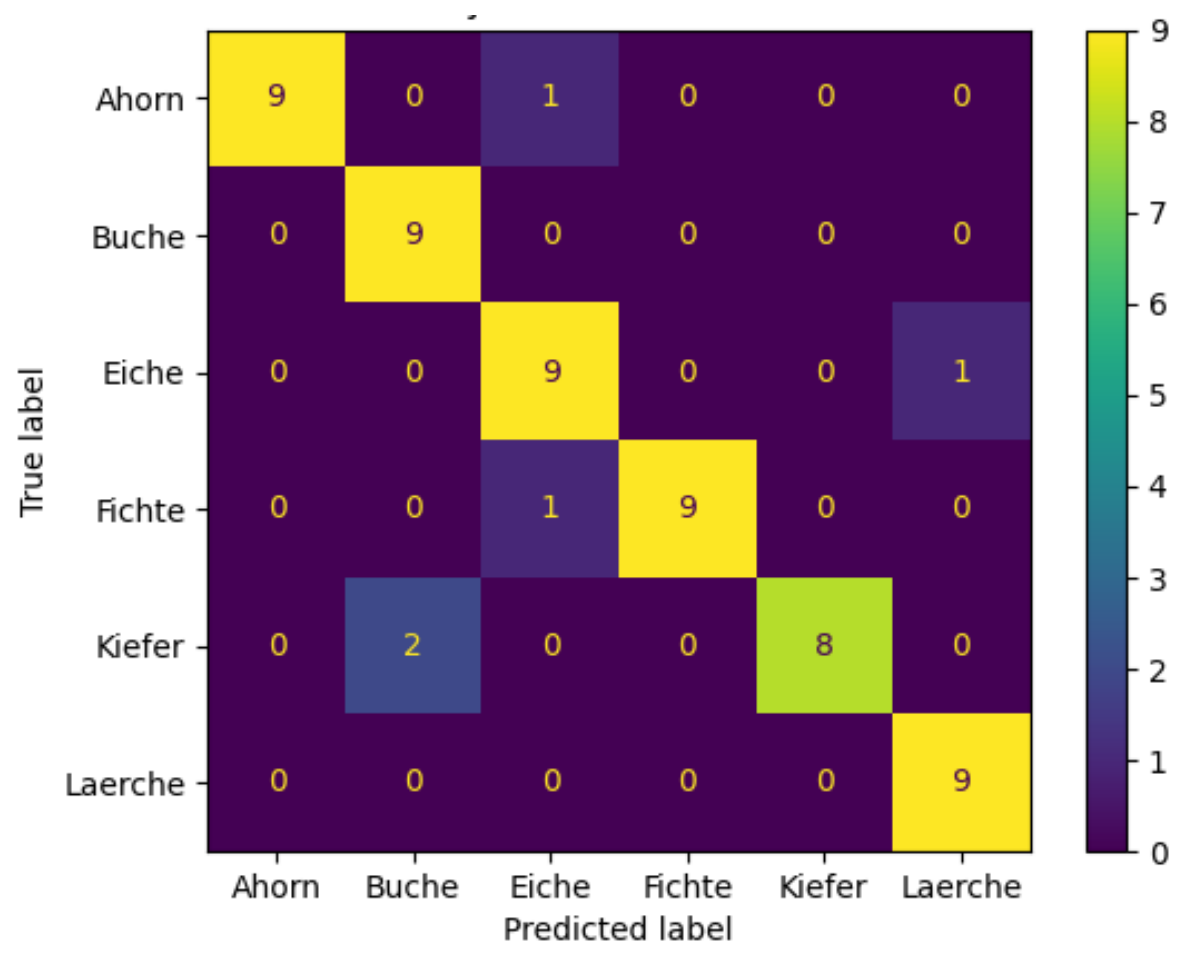

Abbildung 6: Konfusionsmatrix der datenbasierten Klassifizierung von Holzarten bei einer Anregung von $488 \mathrm{~nm}$

Die Diagonale der Konfusionsmatrix zeigt, dass bei einer Anregungswellenlänge von 488nm die Holzart für 53 von 58 Proben korrekt bestimmt wurde. Dies entspricht einer Identifikationsgenauigkeit 
von 91,38\%. Buche und Lärche wurden jeweils 9 von 9-mal richtig erkannt und schnitten in diesem Test am besten ab. Die Identifikation der Holzarten Ahorn, Eiche und Fichte wurden jeweils einmal nicht erkannt. In zwei Fällen wurde Kiefer fälschlicherweise Buche zugeordnet.

\subsection{Fazit}

Die durchgeführten Tests zeigen zwei sehr unterschiedliche Ergebnisse für die verwendeten neuronalen Netze. Die bildliche Klassifizierung unter Verwendung eines CNN konnte mit Ergebnissen unter 50 \% bis jetzt noch nicht die gewünschten Ergebnisse erzielen. Dennoch ist das Potenzial der Bildklassifizierung vorhanden. So könnten durch Erweiterungen des standardisierten Probensatzes und Bereinigung der Daten bessere Ergebnisse erreicht werden. Das Augenmerk sollte jedoch auf die datenbasierte Klassifizierung gerichtet werden. Mit einer Anregungswellenlänge von $405 \mathrm{~nm}$ wurden 57 von 58 Holzproben richtig zugewiesen, was einem Ergebnis von 98,28 \% entspricht. Hier können die gewählten Parameter noch ausgearbeitet werden, da bis jetzt lediglich die Standardabweichung und Mittelwert der gesamten Matrix verwendet wurden. Die Testergebnisse der datenbasierten Klassifizierung der Holzart bei einer Anregungswellenlänge von $405 \mathrm{~nm}$ sind sehr vielversprechend und zeigen ein hohes Potenzial der FD-FLIM Technik für die Holzindustrie. Das Ergebnis soll mit größeren Datensätzen weiter evaluiert werden.

\section{Zusammenfassung und Ausblick}

Eine sortenreine Sortierung für die Weiterverarbeitung von Altholz spielt für einen ressourcenschonenden Umgang eine wichtige Rolle. Derzeit wird das Altholz durch Sichtkontrolle sortiert. Eine zuverlässige und automatisierte Technik konnte noch nicht eingeführt werden. Die Altholzsortierung soll in Zukunft automatisiert, präzise und fehlerfrei funktionieren, um eine optimale Verwendung des Altholzes zu gewährleisten. Daher wurde die Identifikation von Holzarten mittels FD-FLIM und neuronalen Netzwerken analysiert. Es wurden zwei neuronale Netze für die Holzartidentifikation entwickelt und an 6 Holzarten getestet. Die Ergebnisse zeigen, dass die Bildklassifizierung mittels CNN noch nicht für eine solide Identifikation von Holzarten geeignet ist. Eine datenbasierte Klassifizierung mittels MLP ist mit 98,28 \% hingegen äußerst erfolgsversprechend. Die Ergebnisse zeigen, dass eine Anregungswellenlänge von $405 \mathrm{~nm}$ für die Identifikation von Holzarten besonders geeignet ist. Die Ergebnisse bestätigen somit, dass ein hohes Potenzial der Altholzsortierung mittels FD-FLIM in Kombination mit KNN vorhanden ist. Die Unterscheidung verschiedener Holzarten mittels neuronalen Netzes und FD-FLIM soll weiter ausgebaut und optimiert werden.

\section{Literatur}

[1] Meinlschmidt, Peter; Berthold, Dirk; Briesemeister, Robert: Neue Wege der Sortierung und Wiederverwertung von Altholz. In: Recycling und Rohstoffe, Band 6, 2013, S. 153-176

[2] Verordnung über Anforderungen an die Verwertung und Beseitigung von Altholz (Altholverordnung - AltholzV) (BGBl. I S. 3302), zuletzt geändert durch Art. 62 G v. 29.3.2017

[3] Mauruschat, D.; Plinke, B.; Aderhold, J.; Gunschera, J.; Meinlschmidt, P.; Salthammer, T.: Application of nearinfrared spectroscopy for the fast detection and sorting of wood-plastic composites and waste wood treated with wood preservatives. In: Wood Sci Technol 50, 2016, S. 313-331. DOI: 10.1007/s00226-015-0785-x

[4] Hasan, R.; Solo-Gabriele, H.; Townsend T.: Online sorting of recovered wood waste by automated XRFtechnology: part II. Sorting efficiencies. In: Waste management, 31, 4, 2011a, S. 695-704. DOI: 10.1016/j.wasman.2010.10.024. 
[5] Leiter, N.; Wohlschläger, M.; Auer, V.; Versen, M.; Laforsch, C.: A novel approach to identify wood species optically using fluorescence lifetime imaging microscopy. Sensor and Measurement Science International Digital Conference 2021, AMA Service GmbH, ISBN: 978-3-9819376-4-0 , S. 169 - 170.

[6] Valeur, B.; Berberan-Santos, M.: Moleculare Fluorescence. WILEY-VCH Verlag, Weinheim 2012

[7] Schmitz, Julian: Grundlagen Neuronaler Netze. Fakultät für Informatik, Technische Hochschule München

[8] Hope, Tom; Resheff, Yehezkel S.; Lieder, Itay: Einführung in Tensorflow. O’Reilly Verlag, Mai 2018

[9] Brownlee, Jason: Gentle introduction to scikit-learn a python machine learning library. Python Machine Learning, 2014

[10] Steinwendner, Joachim; Schwaiger, Roland: Neuronale Netze programmieren mit Python. 2. Aufl., Rheinwerk. Bonn 2019

[11] IBM Cloud Education: Convolutional neural networks. Online: https://www.ibm.com/cloud/learn/convolutional-neural-networks, 2020

[12] Pandey, Shivangi: Perceptron (Multilayer) Neural Network Algorithm. Online: https://pianalytix.com/perceptronmultilayer-neural-network-algorithm, Pianalytix Edutech Pvt Ltd, 2020 